\title{
Challenges and perspectives of Transmission Kikuchi Diffraction for nanocrystalline materials characterization
}

\author{
Alice Fanta \\ DTU Nanolab, Copenhagen, Denmark
}

Transmission Kikuchi diffraction (TKD) in the scanning electron microscope is maturing as an alternative technique to investigate crystal orientation of nanocrystalline materials. The improved spatial resolution in comparison to electron backscatter diffraction (EBSD) enables revealing the missing details of some microstructure, and finally achieve high-resolution orientation maps of nanocrystalline materials. In the few past years, several applications of the technique were demonstrated ${ }^{1-3}$ and some hardware developments ${ }^{4,5}$ to improve the speed and quality of the data acquired were proposed. Furthermore, studies focusing on for example spatial resolution ${ }^{6}$, depth resolution ${ }^{7,8}$, diffraction contrast $^{9}$ and comparing TKD with precession electron diffraction (SPED) in the TEM were presented ${ }^{10,11}$

As the interest in applying TKD for nanocrystalline orientation mapping increases, it becomes important to discuss some of its challenges. Although one of the biggest advantages of TKD in comparison to TEM based orientation mapping is the easier access to such system, high resolution orientation mapping of nano-structure materials in the SEM can still be very challenging. Some of these challenges are: charging, drift, carbon contamination spatial resolution and depth resolution. In this presentation, we will first focus on describing these challenges and present solutions to reduce/avoid them and obtain high-resolution TKD mapping of nanoparticles. For example, by cleaning the sample with hydrogen plasma we were able to significant reduce carbon contamination of $\mathrm{Cu}$-nanoparticles without oxidizing the particles, which was the case with air plasma. Moreover, we will demonstrate a solution to improve the lateral spatial resolution of TKD by performing orientation maps in immersion mode. And finally, present the perspectives of blocking the saturated signal of the transmitted beam on the on-axis TKD detector to obtain diffraction patterns containing high contrast Kikuchi band and spot patterns simultaneously.
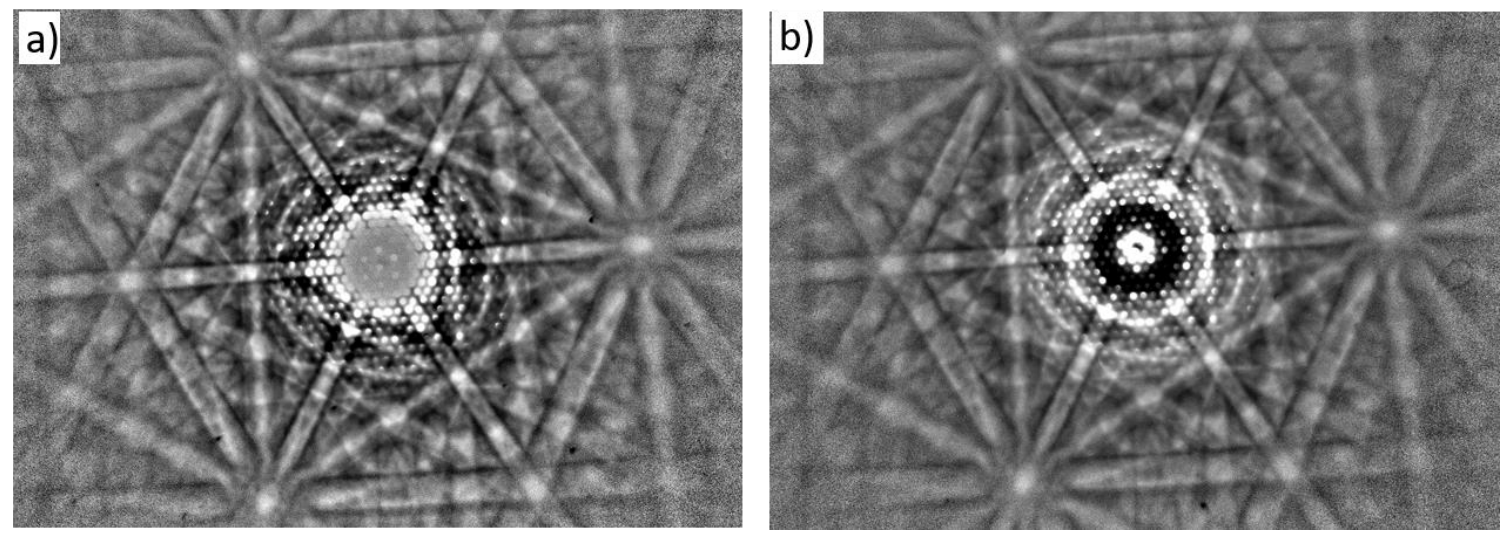

Figure 1. High resolution transmission Kikuchi pattern obtain with standard on-axis TKD detector a) and with post-scintillation filter $b$ ).

References

1. Alekseeva, S. et al. Nat. Commun. 8, 1084 (2017).

2. Daly, L. et al. Geochim. Cosmochim. Acta 216, 42-60 (2017).

3. Lederer, M. et al. Appl. Phys. Lett. 115, 222902 (2019). 
4. Fundenberger, J. J. et al. Ultramicroscopy 161, 17-22 (2016).

5. Fanta, A. B. S. et al. Ultramicroscopy 206, 112812 (2019).

6. Niessen, F., Burrows, A. \& Fanta, A. B. da S. Ultramicroscopy 186, 158-170 (2018).

7. Liu, J., Lozano-Perez, S., Wilkinson, A. J. \& Grovenor, C. R. M. Ultramicroscopy 205, 5-12 (2019).

8. Brodu, E., Bouzy, E. \& Fundenberger, J.-J. Ultramicroscopy 181, 123-133 (2017).

9. Sugar, J. D., McKeown, J. T., Banga, D., \& Michael, J. R. Microscopy and Microanalysis, 1-11(2020). 